\title{
HREM CHARACTERIZATION OF STRUCTURAL CHANGES IN A DEFORMED $\Sigma=9$ (122) GRAIN BOUNDARY IN SILICON
}

\author{
J.L. PUTAUX and J. THIBAULT-DESSAUX \\ Centre d'Etudes Nucléaires de Grenoble, Département de Recherche \\ Fondamentale, Service de Physique, Groupe Structures, 85X, F-38041 \\ Grenoble Cedex, France
}

RESUME: D'importantes modifications structurales ont lieu en déformant, à températures relativement élevées $\left(\mathrm{T}>1020 \mathrm{~K}=0.6 \mathrm{~T}_{f}\right)$, le joint $\Sigma=9(122)$ parfait. Le coeur des dislocations élémentaires de $\Sigma=9$, produites à faible déformation, ainsi que la structure des joints de coincidence d'angles supérieurs, produits à forte déformation, sont décrits grâce au modèle des unités structurales. On constate que la prédiction de 1 'évolution structurale du joint n'est pas immédiate et que le mélange d'unités structurales dépend des conditions de déformations.

ABSTRACT: At relatively high temperature $\left(T>1020 \mathrm{~K}=0.6 \mathrm{~T}_{\mathrm{m}}\right)$, the deformation of a perfect $\Sigma=9(122)$ symmetrical grain boundary (GB) induced large changes in the initial structure. The structural unit model was used to describe the structure of elementary $\Sigma=9$ DSC dislocations cores, produced at low strain, and higher tilt angle coincidence GB's, produced at large strain. It was shown that the prediction of the GB structural evolution was not so straightforward and that the mixing of structural units depended on the deformation conditions.

\section{1 - INTRODUCTION}

One way to describe the structure of tilt grain boundaries (GB's) is to use the structural unit (SU) model developped by SUTTON and VITEK /1/. In the case of cubic diamond material, starting from the early work of HORNSTRA $/ 2 /$, various authors have proposed models to describe different $<011>$ tilt GB's in a large misorientation range and calculated their energy $/ 3,4,5,6 /$. On an other hand, observations by high resolution electron microscopy (HREM) have been performed on Czochralski grown bicrystals with different coincidence $\Sigma$ GB's 17/. They confirmed the validity of the structural units description but emphasized the difficulty of a priori describing particular GB's, revealing for instance a splitting tendancy. The aim of this paper is to investigate the structural changes undergone by a $\Sigma=9(122)$ tilt GB under plastic deformation at different conditions (strain and temperature). Atomic descriptions of the GB's and some of their defects are proposed, using a structura] unit formalism. The possibilities and limitations of the concept of SU mixing are discussed.

\section{2 - EXPERIMENTALS}

Czochralski grown silicon bicrystals, containing a dislocation free symmetrical $\Sigma=9(\overline{1} 2 \overline{2})$ tilt grain boundary $\left(\theta=38.94^{\circ}\right)$, were macroscopical1y deformed by $A$. JACQUES and A. GEORGE at the Ecole des Mines de Nancy (France). A symmetrical compressive stress was applied to the samples. After deformation, specimens were cut, ion thinned and observed in a JEOL $200 C X$ $(200 \mathrm{kV})$ microscope. Imaging conditions suitable for HREM were achieved by putting the common [011] tilt axis of the bicrystal parallel to the electron beam, so that the interfacial structure and its defects could be viewed end-on (for more details, see /8/). In the following, the grain $I$ will be the reference for the indexation of planes and directions.

Experimental micrographs have enabled us to propose atomic models to describe the structure of some observed $\Sigma$ GB's. These models were input in a computer relaxation program which calculated the static (i.e. at $0 \mathrm{~K}$ ) energy of the relaxed configuration. KEATING's interatomic potential was used. Two values of the energy were calculated depending on the set of parameters chosen for the potential, either the KEATING's one /9/ or the BARAFF's one /10/.

3 - RESULTS

In the following, defects and boundary structures will be described with respect to the initial perfect $\Sigma=9 \mathrm{~GB}$ whose model corresponds to the zig zag stacking of the mirror related 

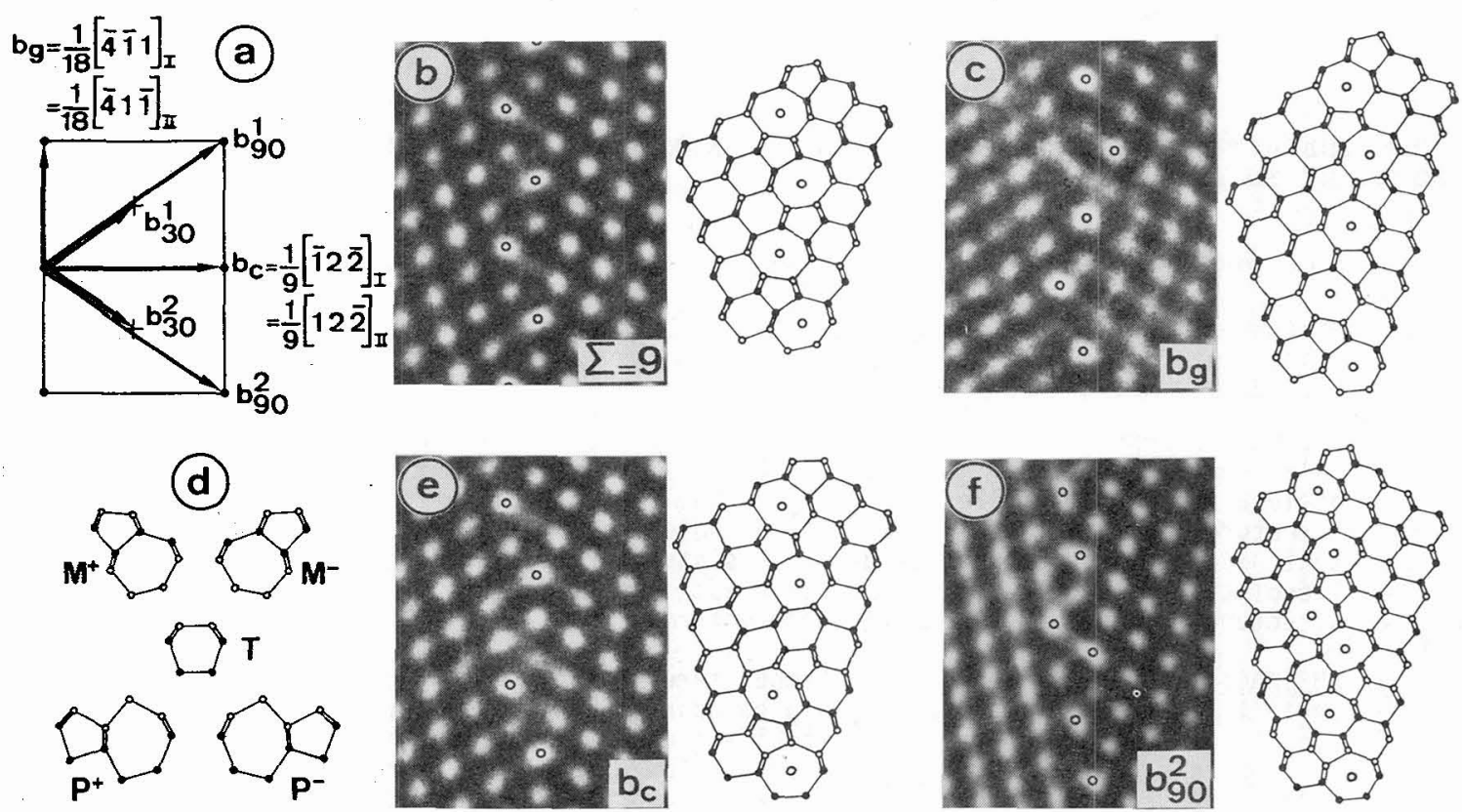

Fig.1 : a) $\Sigma=9$ DSC lattice with vectors of elementary GBD's, ( $0: z=0$ and $+: z=a / 4[011])$, b) initial $\Sigma=9$ GB (black atoms) and its zig-zag $\mathrm{M}^{+} M^{-}$model, d) basic SU's used to describe GB's and defects structures, c) e) and f) micrographs (black atoms) of $\Sigma=9$ GBD's without screw component and corresponding models : c) $b_{g}\left(M^{-}\right)$, e) $b_{c}(T)$ and f) $b_{90}^{2}\left(M^{-} P^{*}\right)$.

$\mathrm{M}^{+}$and $\mathrm{M}^{-}$units (see fig.1b). This description is different from the one based on the stacking of Lomer dislocation cores $/ 2 /$ but, as explained by VAUDIN et al. $/ 4 /$, is suitable to propose models of GB's in the misorientation range of $38.94^{\circ}(\Sigma=9) \leqslant \theta \leqslant 70.53^{\circ}(\Sigma=3)$.

\subsection{Low deformation $(\epsilon=1.7 \%)$; medium temperature $(T=1120 \mathrm{~K})$ :}

The interaction between grain dislocations and a $\Sigma=9$ GB was examined by HREM by EL KAJBAJI et a)./11/ in a sample deformed at $1120 \mathrm{~K}$ up to $\epsilon=0.3 \%$. They showed that a $60^{\circ}$ dislocation was absorbed by the interface where it decomposed, by climb and glide, in three GB dislocations (GBD's) whose Burgers vectors (BV's) were primitive translations of the $\Sigma=9$ DSC lattice : $b_{60}^{1}=b_{c}+b_{9}+b_{30}^{1-}$. At very low deformation $(0.3 \%)$, isolated $G B D$ 's could be well characterized and their cores studied with respect to the $\Sigma=9 \mathrm{~GB}$ structure.

At the same temperature but for a $1.7 \%$ strain, we observed that the interface contained an inhomogeneous distribution of various DSC dislocations /12/. In this paper, we will concentrate our attention on GBD's introducing only rotations and/or shears perpendicular to the [011] tilt axis. Dislocations with a screw component will not be considered here.

$b_{g}$ (and $-b_{g}$ ) : The Bv of this GBD lies parallel to the GB plane. Thus, $b_{g}$ moves along the $\mathrm{GB}$ by pure glide. Its core can be described by the addition of a supplementary $\mathrm{M}^{-}$unit to the initial $\Sigma=9 G B$ (fig.1c), inducing a step whose height is $-h_{0}=-a / 3$. Then the SU sequence is.$M^{+} M^{-} M^{-} M^{+} M^{-} \ldots$ The $-b_{g} G B D$ is mirror related to $b_{g}$. Its core is associated to one $M^{+}$ unit and $a+h_{0}$ step. $b_{g}$ type GBD's do not change the misorientation angle but their motion produces intergranular sliding and lateral migration of the GB. 

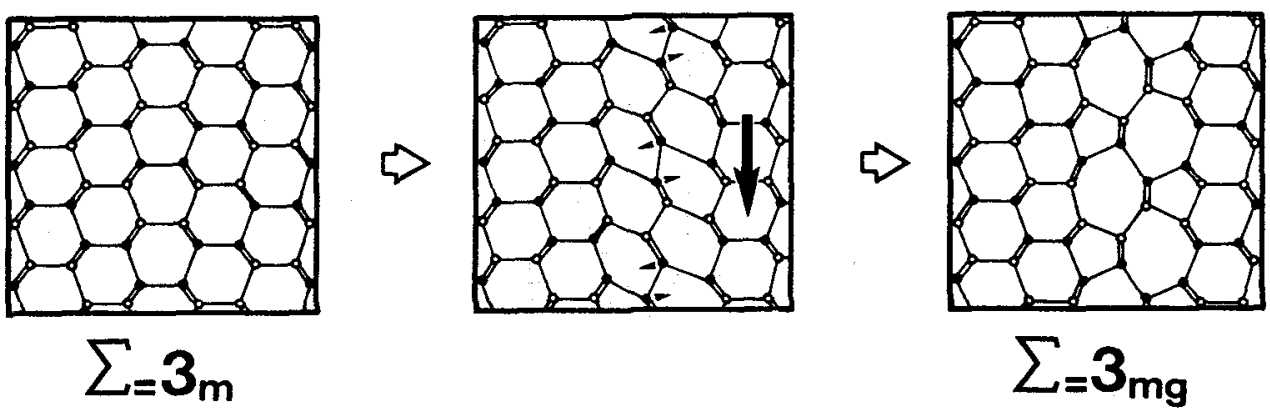

Fig.2 : One way to obtain the $\Sigma=3_{\mathrm{mg}}$ model (with $\mathrm{P}$ units) by applying to $\Sigma=3_{\mathrm{m}}$ (with $\mathrm{T}$ units) a a/6[21T] in grain II parallel to the GB plane (arrows indicate the tilting of atomic rows before a suitable bond reconstruction).

b : With its Bv normal to the boundary plane, $b_{c}$ moves along the GB by pure climb. It is not associated with a step and its core is characterized by a boat shaped 6 -atom ring ( $T$ unit) inserted between two $M$ units of one $\Sigma=9$ period (see fig.l.e). T is the basic SU of the first order twin $\Sigma=3(\overline{1} 1 \overline{1})$. As $T$ introduces a a/4[011] shift, the complete sequence of one $\Sigma=3$ period is $(T T)$. Introduced in $\Sigma=9$, the $T$ unit will be deformed. The two reasons are: i) $\Sigma=9$ and $\Sigma=3$ GB's have different tilt angles giving rise to rotation incompatibilities between $M$ and $T$ units. The $T$ unit induces a $\Delta \theta$ increase in the $\Sigma=9 \mathrm{~GB}$ misorientation angle. ii) $\Sigma=9^{\circ}$ and $\Sigma=3 \quad \mathrm{~GB}^{\prime} \mathrm{s}$ have different "translation states". $\Sigma=3$ has a mirror symmetry and $T$ unit is symmetrical with respect to the (I1I) boundary plane. Because of the zig-zag structure of the mirror glide $\Sigma=9 \mathrm{~GB}$, the T unit will be sheared parallely to the GB plane when inserted between $M$ units. Two sequences are possible: .. $M^{+} M^{-} M^{+} T M^{-} M^{+} M^{-} \ldots$ or . . $M^{+} M^{-} T M^{+} M^{-} M^{+} M^{-} \ldots$. They are mirror related. Thus, in both cases, $T$ units undergo equal and opposite shear stresses but are associated with the same $b_{c} B v$.

$b_{90} \quad:$ Unlike $b_{c}$ and $b_{g}, b_{90}$ is not an elementary DSC dislocation. Its Bv is equal to $b_{c} \pm b_{g} \quad\left(b_{90}^{1}=b_{c}+b_{g}\right.$ and $\left.b_{90}^{2}=b_{c}+\left(-b_{g}\right)\right)$ and this decomposition immediately gives the climb and glide components of the defect. As shown in fig.If, its core can be modelized by using a group of two SU's, noted $M^{+} P^{-}$for $b_{90}^{1}$ and $M^{-} P^{+}$for $b_{90}^{2}$ (the two different cores are mirror related).

$P$ is a SU of a new type corresponding to a different arrangement between two rings of 5 and 7 atoms. In the same way as $T$ is the basic unit of the mirror $\Sigma=3$ GB (noted $\Sigma=3_{m}$ ), $P$ can be used to describe a new model of the first order twin, whose period is then $\left(P^{+} P^{-}\right)$, as shown in fig.2. This stucture has a mirror glide symmetry (the glide vector being a/3[2]1] $+a / 4[011])$, but grains I and II are stil] mirror related. Fig. shows that one possibility to obtain this new model (noted $\Sigma=3_{\mathrm{m}}$ ) is to apply to grain II of the $\Sigma=3$ model a a/6[211] shear parallel to the $\mathrm{GB}$ plane. The reconstruction of distorted bonds illustrated in fig. 2 results in the $\Sigma=3$ model.

The geometrical construction of this model is not difficult however, the question of the $\Sigma=3$ mechanical stability still remains. Computer calculations performed on a relaxed model give a static energy which is very high (see table 1) compared to the one, calculated null (the potential is cut off before the second neighbours), of the $\Sigma=3$ GB. This is clearly in agreement with the fact that this last structure is the only one observed in diamond cubic materials.

\subsection{Low deformation $(\epsilon=1.7 \%)$; high temperature $(T=1470 \mathrm{~K})$ :}

We observed that the GB contained only one type of GBD: $b_{c}$. These defects were homogeneously distributed and formed a more or less regular sub grain boundary superimposed to the original $\Sigma=9 \mathrm{~GB}$. EL KAJBAJI et al./11/ have shown that reactions between GBD's had to be invoked to explain this configuration. During a deformation at high temperature, diffusion is large. One can expect dislocation mobility to be enhanced so that GBD's can interact and react. For instance, two $60^{\circ}$ dislocations coming from both grains and decomposed in 
$b_{60}^{1}=b_{c}+b_{g}+b_{30}^{1-}$ and $b_{60}^{11}=b_{c}+\left(-b_{g}\right)+b_{30}^{2+}$ can react in the following way: $b_{g}+\left(-b_{g}\right)=0$ and $b_{30}^{1-}+b_{30}^{2+}=b_{c}$ (attractive). The net result is : $b_{60}^{1}+b_{60}^{11}=3 b_{c}$. Then, if the deformation conditions are symmetrical (i.e. the numbers of dislocations coming from both grains are equal), it's possible to obtain several $b_{c}$ 's that will move in the GB to be evenly distributed and minimize the elastic energy.

\subsection{Large deformation:}

Two samples were deformed up to a $6.8 \%$ strain, at $1220 \mathrm{~K}$ and $1470 \mathrm{~K}$, resulting in a 7 arge increase in the macroscopic misorientation angle of the bicrystal: $\theta \simeq 51^{\circ}$. However, due to a highly heterogeneous deformation, lower and larger misorientation angle areas, separated by sub grain boundaries in the grains $/ 13 /$, were observed, at nanoscopic scale, along the same interface. We will particularly pay attention here to sections of the GB exhibiting periodical structures with only few defects.

$\Sigma=187(\overline{5} 9 \overline{9})[011]-\theta=42.88^{\circ}(\mathrm{T}=1220 \mathrm{~K})$ :

Half a period of this $G B$ is obtained when one $T$ unit is introduced in two $\Sigma=9$ periods, resulting in the sequence: $\left(\mathrm{M}^{+} \mathrm{TM}^{-} \mathrm{M}^{+} \mathrm{M}^{-}\right)$. Because of the $\mathrm{a} / 4[011]$ shift due to $\mathrm{T}$, the complete $\Sigma=187$ period is twice the previous sequence. This model is built following the idea that minority units $(T)$ have to be evenly distributed among majority ones $(M)$. As al ready shown in $\$ 2.1 ., T$ can be $p 1$ aced between either $M^{+}$and $M^{-}$, or $M^{-}$and $M^{+}$. This leads to two possible mirror related variants for $\sum=187$ period: $\left(M^{+} T M^{-} M^{+} M^{-}\right) \times 2$ or $\left(M^{-} T M^{+} M^{-} M^{+}\right) \times 2$. Both structures are energetically equivalent (see table 1). However, only a study of these configurations energy could help to actually decide if the equilibrium state of this $G B$ is described by either one of the two models or a convenient mixture of both. Nevertheless, we observed areas of both types separated by defects. Fig.3a shows such an example where one $\mathrm{M}^{+}$ unit in missing in the original sequence. On each side of the defect, one can observe the two $\Sigma=187$ variants.

\section{$\sum=11(\overline{2} 3 \overline{3})[011]-\theta=50.48^{\circ}$ :}

- medium temperature $(1220 \mathrm{~K})$ : When two $T$ units are introduced per one $\Sigma=9$ period, $M$ and $T$ units are in equal proportions. The distribution is homogeneous when one $T$ separates two $M$ units. The periodical sequence $\left(M^{+} T M^{-} T\right)$ corresponds to a mirror glide zig zag model of the $\Sigma=11 \mathrm{~GB}$ and was observed in our specimen (noted $\Sigma=11$ in fig.3b).

- high temperature $(1470 \mathrm{~K})$ : The pictures exhibit a different structure, similar to the one already detected by BOURRET et a1. in as-grown bicrystal $/ 7 \%$. The period is doubled, with respect to the previous case, and the proposed model now includes a mixture of SU's associated with the $b_{c}$ and $b_{90} \sum=9$ GBD's. The sequence is $\left(M^{+} T M^{-} P^{+} M^{-} T M^{+} P^{-}\right)$and has a mirror glide symmetry, but grains I and II are mirror related (the model is noted $\Sigma=11_{B}$ in fig.3c). We have to point out that calculations performed on both models lead to very static energies (absolute and relative values depending on the chosen potential. See table 1).

$\Sigma=41(\overline{3} 4 \overline{4})[011]-\theta=55.88^{\circ}(T=1220 \mathrm{~K})$ :

If the $G B$ absorbs more dislocations, the misorientation angle keeps increasing. If we extrapolate the previous examples $\left(\Sigma=187\right.$ and $\left.\Sigma=11_{A}\right)$ and introduce more $T$ units in the $G B$ sequence, a possible model of $\Sigma=41 \mathrm{~GB}$ is: $\left(M^{+} T T M^{-} T T\right)$. This time, $M$ are the minority units, evenly distributed among the majority $T$ units. The model has a mirror glide symmetry and a zig zag structure (see fig.3d).

Experimentally, we observed GB zones with a periodicity compatible with a $\Sigma=41$ coincidence. Owing to a small twist component, one has to be very cautious to analyse the pictures. Nevertheless, it appeared that the previous model was not suitable to describe the micrograph of figure $3 d$. Instead, we proposed another model whose sequence is noted: $\left(M^{+} P^{-} P^{+} M^{-} T T\right)$ or $\left(M^{-} P^{+} P^{-} M^{+} T T\right)$. The first model of $\Sigma=41$ contains two periods of $\Sigma=3 \mathrm{mB}$, separated by $M$ units and undergoing equal and opposite shears, whereas the observed structure now includes one period of each $\Sigma=3$ model presented in \$2.1. and has a different translation between both grains. Comparing the shear distributions along the two SU stackings, one can see that, in the new moder, each $\Sigma=3$ period is closer to its "ideal " (i.e. undistorted) state. Moreover, once again, close static energies are calculated for both SU sequences (table 1 ). 

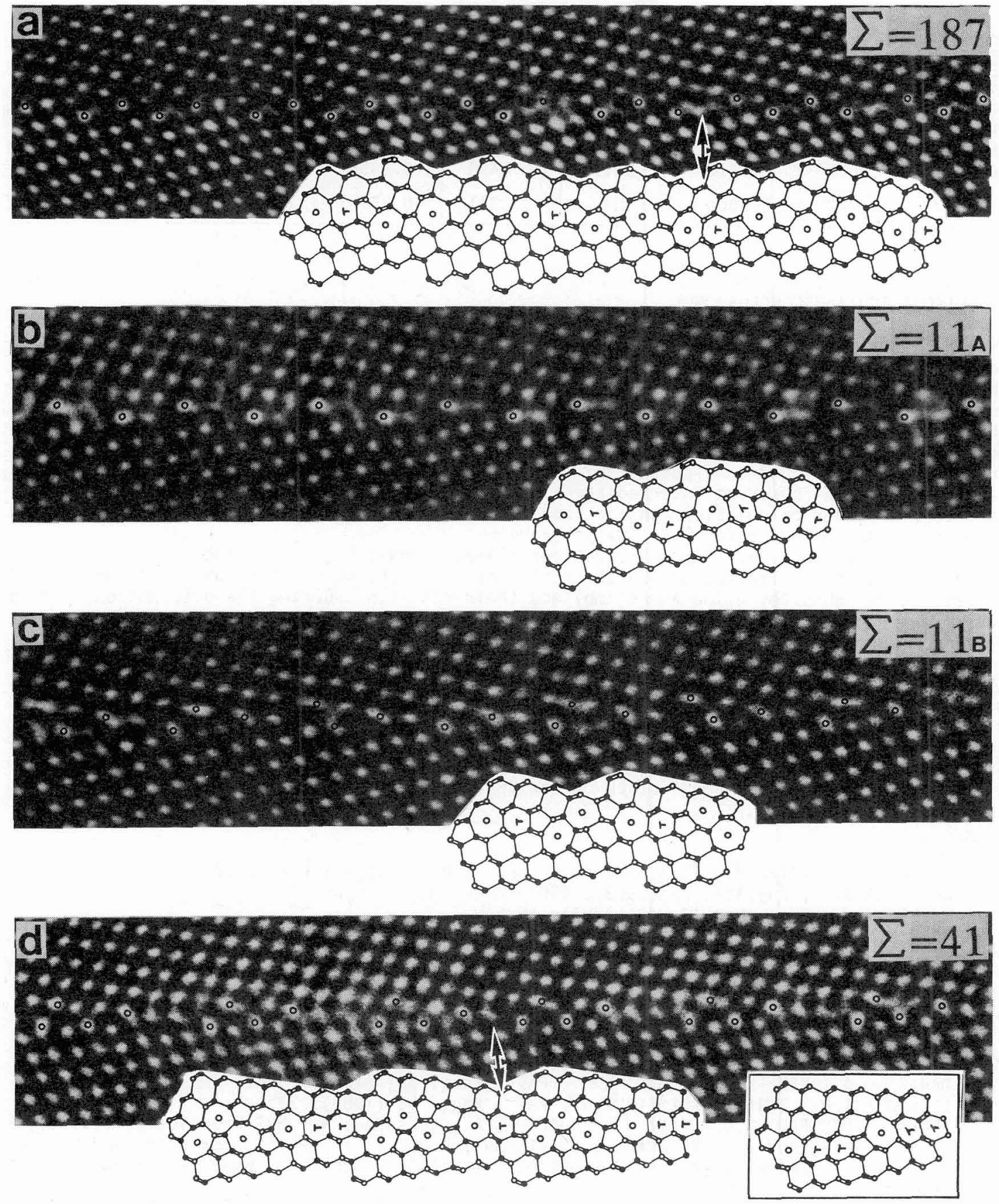

Fig.3 : Micrographs (black atoms) of coincidence GB's obtained by deformation of the initial $\Sigma=9$ and their atomic mode1. a) $\Sigma=187$ : a defect corresponding to a missing $M$ unit (indicated by the arrow) separates two mirror related variants. b) $\Sigma=11$ (obtained at medium temperature) with a simple period containing only $M$ and $T$ units. c) $\Sigma=11_{B}$ (obtained at high temperature) with a double period containing $M, T$ and $P$ units. d) $\Sigma=41:$ model with $M$, $T$ and $P$ units; a defect corresponding to a missing $T$ unit is indicated by an arrow. The non observed mirror model is given for comparison in the frame. 
Table 1. : Values of the static energy for different $\Sigma$ GB, calculated on models relaxed with KEATING's potential. Two sets of parameters are used.

\begin{tabular}{|c|c|c|c|c|c|c|c|c|}
\hline$\gamma \mathrm{J} . \mathrm{m}^{-2}$ & $\Sigma=9$ & $\Sigma=187$ & $\Sigma=11_{\mathrm{A}}$ & $\Sigma=11_{\mathrm{B}}$ & $\Sigma=41_{\mathrm{A}}$ & $\Sigma=41_{\mathrm{B}}$ & $\Sigma=3_{\mathrm{m}}$ & $\Sigma=3_{\mathrm{mg}}$ \\
\hline Keating & 0.6027 & 0.7610 & 0.8041 & 0.8493 & 0.8356 & 0.8669 & 0. & 1.0868 \\
Baraff & 0.2222 & 0.3006 & 0.3285 & 0.3311 & 0.3570 & 0.3397 & 0. & 0.3996 \\
\hline
\end{tabular}

\section{4 - DISCUSSION and CONCLUSION}

From a theoretical point of view, the structural evolution of the initial GB from $\Sigma=9$ to $\Sigma=3$ could be continuously described in a very simple manner, by mixing SU's of these two low energy short period delimiting GB's /15/. By incorporating more and more $T$ units of $\Sigma=3$ (minority units) in the original $\Sigma=9$, one would transform progressively this $G B$ into a $\Sigma=3$ where the M units of $\Sigma=9$ are in minority.

However, we proposed new models to account for the HREM micrographs. The $P$ unit was introduced which was found - a posteriori - to describe an unfavoured high energy $\Sigma=3 \mathrm{~GB}$. The sequences of the observed intermediate $\Sigma$ were built with a mixture of three kinds of SU's. Moreover, computer calculations on the two different models (with or without $P$ units) of the same coincidence GB Ted to close static energies.

As a matter of fact, our HREM observations clearly showed that the structural evolution of the interface was controlled by the absorption of the incoming dislocations, the movement of the resulting GBD's (by glide and $\mathrm{climb}$ ) and their reactions. During the deformation, the GB passes through various intermediate states which depends on the internal stresses and are far from equilibrium. At a sufficiently high temperature these configurations can relax, leading to periodical structures on more or less wide areas.

Three types of structural units can now be used to build various possible reconstructed models of coincidence GB's from $\Sigma=9$ to $\Sigma=3$. Then, if static calculations allow one to put aside models with a too prohibitive energy, they cannot help decide - a priori - which of the remaining ones will be favoured. In the present case, the problem remains if the observed mixings of three SU's (M, T and P) are stabilized by local stresses conditions or if they ensure the global equilibrium of the $G B$ at a given temperature.

\section{REFERENCES:}

1/ SUTTON A.P., VITEK V., Phil. Trans. R. Soc. Lond., A309 (1983), 1-36

12/ HORNSTRA J.R., Physica, 25 (1959), 409

/3/ MOLLER H.J., Phil. Mag. A, 43 (1981), 1045

14/ VAUDIN M.D., CUNNINGHAM B., AST D.G., Scripta Met., 17 (1983), 191

15/ PAPON A.M., PETIT M., Scripta Met., 19 (1985), 391

16/ KOHYAMA M., YAMAMOTO R., DOYAMA M., Phys. Stat. Sol. (b), 137 (1986), 11

77) BOURRET A., BACMANN J.J., Rev. Phys. Appl., 22 (1987), 563

/8/ EL KAJBAJI M., THIBAULT-DESSEAUX J., MARTINEZ-HERNANDEZ M., JACQUES A., GEORGE A, Rev. Phys. Appl., 22 (1987), 569

/9/ KEATING P.N., Phys. Rev., 145 (1966), 637

110/ BARAFF G.A., KANE E.O., SCHLUTER M., Phys. Rev., B21 (1980), 5662

/11/ EL KAJBAJI M., THIBAULT-DESSEAUX. J., Phil. Mag., 58 (1988), 325

12) THIBAULT-DESSEAUX J., PUTAUX J.L., JACQUES A., ÉL KAJBAJI M., MRS Proc.Vol.22 (1988), 29

113/ THIBAULT-DESSEAUX J., PUTAUX J.L., KIRCHNER H.0.K., in Point, Extended and Surface Defects in Semiconductors (1988/89) ed. Cavallini, Schröter, Benedek (PJenum Pub. in the press).

14/. THIBAULT-DESSEAUX J., PUTAUX J.L., in Structure and Properties of Dislocations in Semiconductors, Oxford Conf. (1989) in the press.

/15/ SUTTON A.P., Acta Metal1., 36, (1988), 1291. 